\title{
Hydromagnetic Mixed Convection along a Vertical Cylinder in a Stratified Non-Darcian Porous Medium
}

\author{
Saddam Atteyia Mohammad ${ }^{1}$, Abdelkrim Younis Fathi ${ }^{2}$ \\ ${ }^{1,2}$ University of Mosul-College of Engineering-Mechanical Engineering Department \\ Almajmuea 41002, Mosul, Iraq \\ saddamatteyia@uomosul.edu.iq; abd59you@gmail.com
}

\begin{abstract}
In the present problem, the simultaneous heat and mass transfer by steady, laminar, incompressible, viscous and conductive to electricity combined convection flow straight a vertical cylinder placed inside a non-Darcian thermally stratified porous medium are considered. The condition of variable surface temperature and variable surface concentration are analyzed. All the regime of convection (free, mixed, and forced) will be studied. The governing nonlinear partial differential equations will be converted to nonsimilar form by using suitable transformations. Then they are solved by a finite difference method. Hydrodynamic, thermal, and concentration profiles as well as local Nusselt and Sherwood numbers that reflect the amount of heat and mass transfer will be analyzed and discussed. The results show that increasing the value of the power law index of the surface temperature of the cylinder, curvature parameter, and buoyancy ratio leads to enhance the rates of heat and mass transfer. On contrast the rates of heat and mass transfer are reduced when the value of inertia effect parameter, square of the Hartmann number, and local thermal stratification parameter are raised. Enhancing the value of Lewis number causes lowering the rate of heat transfer and growing the rate of mass transfer.
\end{abstract}

Keywords: Vertical cylinder, Heat and Mass transfer, Porous medium, Mixed convection, Thermal stratification

\section{Introduction}

The subject of convective heat transfer from cylindrical geometry has received more attention from the researchers. This is due to the fact that cylinders have been used in chemical catalytic reactors, geothermal systems, nuclear waste disposal, heat exchangers and so on (Chen and Horng [1]). Stratification is a physical phenomenon may be arise from temperature difference, that causes a divergence in the density of the fluid and in this case this process is called as thermal stratification. This is due to the input of thermal energy into the medium by thermal sources or heated bodies. Concentration difference is another situation that cause a stratification like transmit process in the sea arising from salinity distinction. The presence of different fluids may lead to arise of stratification. Stable state emerges when the fluid of light density overlies the fluid of heavy density.

From the examples of convection operations that happen in ambience with stratification phenomenon are environmental chambers with heated walls and closed containers. Another example is the convection flow connected with heat-removal systems for long-period profound ocean power modules. Furthermore, diverse buoyant flow systems inclusive geological transport, geothermal systems, lake thermo hydraulics, condensation systems of power plant, and volcanic flows and also in industrial thermal processing are of great interest (Mukhopadhyay and Ishak [2]).

The Darcy's law is valid only for flows of low velocity through porous medium with low permeability. Including the non-Darcy effects is necessary to model the real situation of flow in porous media that contain convection heat and mass transfer. The non-Darcian effects included boundary viscous resistance, porosity variation close the surface (due to packing of particles), inertia effects, and thermal dispersion (Chen [3]). The boundary viscous resistance may be regarded considerable when heat transfer is treated in a zone very near to the surface. The Brinkman's extension that is used to give weight for boundary viscous resistance, join viscous shear stress term within the equation of momentum as well as the noslip boundary condition. The inertia effects is considered significant at a higher flow rate and it can be taken into consideration through the addition of a velocity squared term (known as the Forchheimer's extension) in the momentum equation. While inertia effects are predominant, the thermal dispersion effect may become significant. Thermal dispersion occur due to recirculation and mixing of local fluid streams as the fluid flows through tortuous paths formed by the solid particles. Thus a good blending of the fluid happens inside the pores (Chen and Horng [1], Afify [4]). 
Researchers has gave good attention to the subject of magneto hydrodynamic MHD and heat and mass transfer in different geometries. The reason of this attention is the influence of magnetic field on the control of flow and execution of many systems that use fluids conductive to electricity. Many metallurgical processes in industry include cooling process of continued strips or filaments via drawing them in fluid conducting to electricity in the existence of magnetic field. From the examples of these processes is the drawing, annealing, and tinning of copper wires. In these processes the rate of cooling can be controlled which can be affect the properties of the final product (Amanull et al. [5]; Ganapathirao et al. [6]). Moreover, in different technological applications such as purification of molten metals, geothermal energy extraction, nuclear reactor coolers, metal casting, and many others the effects of magnetic field are encountered. The fluids in geothermal regions are electrically conducting and can be significantly influenced by the magnetic field. This fact is motivated the study of the impact of magnetic field on the transport phenomenon over porous media (Ganapathirao et al. $[6])$.

The problem of mixed convection flow past a vertical cylinder was investigated by Merkin and Pop [7]. Thermal dispersion effect on the non-Darcy mixed convection flow over a vertical cylinder was investigated by Kumari et al. [8]. Using Darcy model, Hooper et al. [9] study the mixed convection along an isothermal vertical cylinder. The case of nonDarcy combined convection flow over vertical cylinder was investigated by Aldoss et al. [10]. With non-Darcy model, Aldoss [11] in another study considered magneto hydrodynamic mixed convection flow adjacent to a vertical cylinder. Mixed convection flow adjacent to a vertical heated or cooled permeable slender cylinder was investigated by Kumari et al. [12] using the Darcy law in order to examine the impact of steady non-uniform suction or injection. The problem of mixed convection flow over a vertical cylinder embedded in a nanofluid-saturated porous medium is studied by Gorla and Hossain [13]. Steady mixed convection boundary layer flow on a vertical cylinder in a porous medium saturated with a nanofluid for cooled and heated cylinder is studied by Rohni et al. [14].

The case of conjugate heat transfer of magneto hydrodynamic mixed convection flow of nanofluid past a vertical slender hollow cylinder in a high porosity porous medium is analyzed numerically by Jafarian et al. [15]. Shu et al. [16] study the problem of steady mixed convection boundary layer flow on a cooled vertical permeable cylinder. Mohammad [17] investigated the problem of coupled heat and mass transfer by steady mixed convection flow of electrically conducting fluid past a vertical circular cylinder embedded in non-Darcy porous medium with heat source. Mohammad [18] performed analysis to study the influences of variable viscosity on hydromagnetic coupled heat and mass transfer by mixed convection non-Darcy flow adjacent to a vertical circular cylinder embedded in a porous medium.

In this paper, It will be study hydromagnetic simultaneous heat and mass transfer by mixed convection boundary layer flow past a vertical circular cylinder embedded in a stratified non-Darcian porous medium. The entire regime of mixed convection was considered. In other words calculations will be carried out from perspicuous natural convection edge to perspicuous forced convection edge. Numerical modeling is widely used in analyzing of such problems because of its ability of providing specific conception about the physics of problems from this type, furthermore, the experimental work require high cost and time. This work finds applications in petroleum engineering, geothermal systems, insulation technology, and nuclear engineering. To the best knowledge of the authors this problem does not appear in the previous literature, therefore, the obtained numerical results are original and novel.

\section{Problem Formulation}

Consider laminar, incompressible, steady, and hydromagnetic coupled heat and mass transfer by non-Darcian mixed convection flow along a vertical circular cylinder in a thermally stratified porous medium. Figure 1 depicts the physical flow model and coordinate system. Moreover, the following assumptions are made in the formulation of the present problem:

1. The fluid in the medium is Newtonian and electrically conducting.

2. The fluid has constant properties exclude the density of the buoyancy term of the balance of momentum equation.

3. Density is approximated by the Boussinesq approximation.

4. Boundary-layer approximations are applicable.

Under the above assumptions the governing nonlinear equations are given as: 


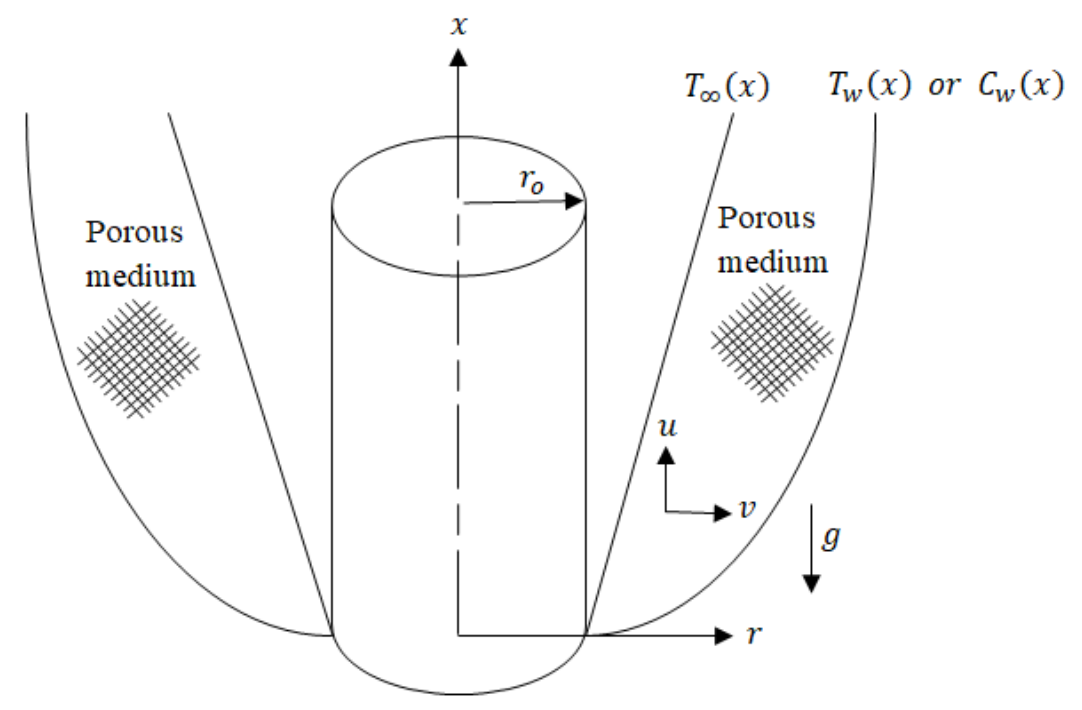

Fig. 1: Physical flow model and coordinate system.

\subsection{Continuity Equation}

$$
\frac{\partial u}{\partial x}+\frac{\partial(r v)}{\partial r}=0
$$

Where $u$ and $v$ represents the components of fluid velocity in the $x$ and $r$ axes respectively.

\subsection{Momentum Equation (Mohammad [17]; Mohammad [18])}

$$
\begin{gathered}
u=\frac{-K}{\mu}\left(\frac{\partial P}{\partial x}+\rho g+\frac{\sigma \beta_{o}^{2} u}{\phi}\right)-\frac{c \sqrt{K} u^{2}}{v} \\
v=\frac{-K}{\mu}\left(\frac{\partial P}{\partial r}\right)-\frac{c \sqrt{K}}{v} v^{2} \\
c=1.75 /\left(\sqrt{150} \quad \phi^{1.5}\right) \quad K=d^{2} \phi^{3} /\left[150(1-\phi)^{2}\right]
\end{gathered}
$$

Where $K$ is the permeability of the porous medium, $\phi$ is the porosity of the porous medium, $d$ is the particle diameter, $c$ is constant, $\sigma$ is the electrical conductivity of the fluid, $\beta_{o}$ is the magnetic induction, $P$ is the pressure, $\rho$ is the density of the fluid, $\mu$ is the dynamic viscosity of the fluid, and $g$ is the gravitational acceleration. It can be eliminate pressure term by differentiating Eq. (2) with respect to $r$ and Eq. (3) with respect to $x$. Apply approximation of the Boussinesq

$$
\rho=\rho_{\infty}\left[1-\beta_{T}\left(T-T_{\infty}\right)-\beta_{C}\left(C-C_{\infty}\right)\right]
$$

Where $T$ and $C$ are temperature and concentration respectively. $T_{\infty}$ and $C_{\infty}$ are free stream temperature and concentration respectively. $\rho_{\infty}$ is the free stream density. $\beta_{T}$ and $\beta_{C}$ are the coefficients of thermal and concentration expansion respectively. As stated earlier, the boundary layer approximations are applicable, thus, within the boundary layer $(v \ll u, \partial v / \partial x \ll \partial u / \partial r)$. According to the above information the final format of the momentum equation can be given by

$$
-\frac{\mu}{K} \frac{\partial u}{\partial r}-\frac{2 c \rho}{\sqrt{K}} u \frac{\partial u}{\partial r}-g \frac{\partial \rho}{\partial r}-\frac{\sigma \beta_{o}^{2}}{\phi} \frac{\partial u}{\partial r}=0
$$




\subsection{Energy Equation}

$$
u \frac{\partial T}{\partial x}+v \frac{\partial T}{\partial r}=\alpha\left[\frac{\partial 2 T}{\partial r^{2}}+\frac{1}{r} \frac{\partial T}{\partial r}\right]
$$

In Eq. (7) $\alpha$ represent the thermal diffusivity.

\subsection{Mass Conservation Equation}

$$
u \frac{\partial C}{\partial x}+v \frac{\partial C}{\partial r}=\frac{D}{r} \frac{\partial}{\partial r}\left(r \frac{\partial C}{\partial r}\right)
$$

In Eq. (8) $D$ represent the mass diffusivity.

\subsection{Boundary Conditions}

$$
\begin{array}{cccc}
r=r_{o} & v=0 & T=T_{w}(x)=T_{\infty, 0}+A x^{m} & C=C_{w}(x)=C_{\infty, 0}+A x^{m} \\
r \rightarrow \infty & u=U_{\infty} & T=T_{\infty}(x)=T_{\infty, 0}+B x & C=C_{\infty, 0}
\end{array}
$$

Where $T_{\infty, 0}$ and $C_{\infty, 0}$ are the free stream temperature and concentration at $x=0$ respectively. The subscript $w$ indicate the condition at the cylinder wall.

\subsection{Dimensionless Variables}

The following dimensionless variables will be introduced to convert the system of governing differential equations into a non-similar form. The non-similar system of equations is applicable from free convection limit through mixed convection limit until reached the forced convection limit.

$$
\begin{array}{ll}
\eta=\frac{1}{x} P e_{x}^{1 / 2} \zeta^{-1}\left(\frac{r^{2}}{2 r_{o}}-\frac{r_{o}}{2}\right) & \zeta=\left[1+\left(\frac{R a_{x}}{P e_{x}}\right)^{1 / 2}\right]^{-1} \\
f=\frac{\psi(\zeta, \eta)}{\alpha r_{o} P e_{x}^{1 / 2} \zeta^{-1}} \quad \theta(\zeta, \eta)=\frac{T-T_{\infty}(x)}{T_{w}(x)-T_{\infty, 0}} & S=\frac{T_{\infty}(x)-T_{\infty, 0}}{T_{w}(x)-T_{\infty, 0}} \quad \Phi(\zeta, \eta)=\frac{C-C_{\infty, 0}}{C_{w}-C_{\infty, 0}}
\end{array}
$$

Where $\eta$ and $\zeta$ are the pseudosimilarity variable and the non-similarity parameter respectively. Value of $\zeta$ ranging from 0 to $1 . \zeta=0$ for pure free convection and $\zeta=1$ for pure forced convection. $\psi$ and $f$ are the stream function and dimensionless stream function respectively. $\psi$ is given by $u=(1 / r)(\partial \psi / \partial r)$ and $v=-(1 / r)(\partial \psi / \partial x)$, thus, the equation of continuity is satisfied. $\theta$ and $\Phi$ are dimensionless temperature and dimensionless concentration respectively. $S$ is the local thermal stratification parameter. $P e_{x}$ is the local Peclet number its equal to $P e_{x}=U_{\infty} x / \alpha$. $R a_{x}$ is the local Rayleigh number its equal to $R a_{x}=\left[g \beta_{T} K x\left(T_{w}-T_{\infty}\right) / \alpha v\right]$. When we substituting the dimensionless variables into momentum equation, energy equation, concentration equation, and boundary conditions the following non-similar boundary layer dimensionless equations can be obtained:

\subsection{Dimensionless Momentum Equation}

$$
\left[1+2 \Gamma f^{\prime}+M\right] f^{\prime \prime}+(1-\zeta)^{2}\left(-\theta^{\prime}-N \Phi^{\prime}\right)=0
$$

Where $\Gamma=\frac{c \sqrt{K}\left(P e_{x}^{1 / 2}+R a_{x}^{1 / 2}\right)^{2} \alpha}{v x}$ is the inertia effect parameter, $M=\frac{\sigma \beta_{o}^{2} K}{\mu \phi}$ is the square of the Hartmann number, and $N=\frac{\beta_{C}}{\beta_{T}}$ is the buoyancy ratio. 


\subsection{Dimensionless Energy Equation}

$-[2 \eta \lambda+1] \theta^{\prime \prime}+\left[-\frac{1}{2}(1+m(1-\zeta)) f-2 \lambda\right] \theta^{\prime}+m f^{\prime} \theta=-\frac{m}{2} \zeta(1-\zeta)\left[\frac{\partial f}{\partial \zeta} \theta^{\prime}-\frac{\partial \theta}{\partial \zeta} f^{\prime}\right]-S m f^{\prime}$

Where $\lambda=\frac{x}{r_{o}} \frac{1}{\left(P e_{x}^{1 / 2}+R a_{x}^{1 / 2}\right)}$ is the curvature parameter. When the value of $\lambda$ is equal to zero, this situation is corresponds to vertical flat plate.

\subsection{Dimensionless Concentration Equation}

$-\frac{1}{L e}(2 \eta \lambda+1) \Phi^{\prime \prime}+\left[-\frac{1}{2}(1+m(1-\zeta)) f-\frac{2 \lambda}{L e}\right] \Phi^{\prime}+m f^{\prime} \Phi=-\frac{m}{2} \zeta(1-\zeta)\left[\frac{\partial f}{\partial \zeta} \Phi^{\prime}-\frac{\partial \Phi}{\partial \zeta} f^{\prime}\right]$

Where $L e=\alpha / D$ is the Lewis number.

\subsection{Dimensionless Boundary Conditions}

$$
\begin{array}{ccrlrl}
f(\zeta, 0) & =0 & \theta(\zeta, 0) & =1-S & & \Phi(\zeta, 0)=1 \\
f^{\prime}(\zeta, \infty) & =\zeta^{2} & \theta(\zeta, \infty) & =0 & & \Phi(\zeta, \infty)=0
\end{array}
$$

The formulas of velocity components, local Nusselt number and local Sherwood number in terms of dimensionless variables can be expressed by:

$u=\frac{U_{\infty}}{\zeta^{2}} f^{\prime}$

$v=-\frac{r_{o}}{r} \frac{\alpha}{x} P e_{x}^{1 / 2} \frac{1}{\zeta}\left[\frac{1}{2}(1+m(1-\zeta)) f-\frac{1}{2}(1-m(1-\zeta)) \eta f^{\prime}-\frac{1}{2} m \zeta(1-\zeta) \frac{\partial f}{\partial \zeta}\right]$

$\frac{N u_{x}}{P e_{x}^{1 / 2} \zeta^{-1}}=-\theta^{\prime}(\zeta, 0)$

$\frac{S h_{x}}{P e_{x}^{1 / 2} \zeta^{-1}}=-\Phi^{\prime}(\zeta, 0)$

It is remind to state that, the presence of $\partial / \partial \zeta$ in the above dimensionless equations makes them non-similar. Also, the primes means partial differentiation with respect to $\eta$.

\section{Numerical Solution procedure}

The condition $\eta \rightarrow \infty$ in Eq. (16) is displaced by $\left(\eta=\eta_{\max }\right)$ where $\eta_{\max }$ is adequately large value so that the condition of velocity is satisfied. The domain of solution $(\zeta, \eta)$ is divided into equal spaced mesh in the $\eta$ direction where $\Delta \eta=0.02$ and another equal spaced mesh in the $\zeta$ direction where $\Delta \zeta=0.1$. To conserve space, the method used to solve the non-similar system of Eqs. (12) to (14) are presented in Mohammad [18].

\section{Results and Discussion}

To assess the accuracy of the numerical results to be presented in the subsequent paragraphs, a compare on special case of the problem with previously published work is conducted. The comparison of local Nusselt number for the whole regime of combined convection with different values of power law index $m$ and for the case of Darcy flow when the curvature parameter is equal to zero $(\lambda=0$ corresponds to vertical wall) is shown in Table 1 . 
Table 1: Comparison of $N u_{x} /\left(P e_{x}^{1 / 2} \zeta^{-1}\right)$ values along vertical wall $(\lambda=0)$ for previously published work against present work at different values of $m$ and $\zeta$ for Darcy flow.

\begin{tabular}{|c|c|c|c|c|c|c|}
\hline & \multicolumn{3}{|c|}{ Hsieh et al. [19] } & \multicolumn{3}{c|}{ Present work } \\
\cline { 2 - 6 }$\zeta$ & $m=0$ & $m=0.5$ & $m=1$ & $m=0$ & $m=0.5$ & $m=1$ \\
\hline 0 & 0.4438 & 0.7704 & 1 & 0.4442 & 0.7708 & 1.0004 \\
\hline 0.1 & 0.4035 & 0.6991 & 0.9071 & 0.4039 & 0.6991 & 0.9069 \\
\hline 0.2 & 0.3732 & 0.6419 & 0.8314 & 0.3736 & 0.6418 & 0.8311 \\
\hline 0.3 & 0.355 & 0.6026 & 0.7783 & 0.3553 & 0.6024 & 0.7779 \\
\hline 0.4 & 0.3506 & 0.5844 & 0.7522 & 0.3506 & 0.584 & 0.7516 \\
\hline 0.5 & 0.3603 & 0.589 & 0.7555 & 0.3603 & 0.5886 & 0.7548 \\
\hline 0.6 & 0.3832 & 0.616 & 0.7877 & 0.3832 & 0.6157 & 0.7872 \\
\hline 0.7 & 0.4174 & 0.6629 & 0.8457 & 0.4174 & 0.6627 & 0.8454 \\
\hline 0.8 & 0.4603 & 0.7259 & 0.925 & 0.4603 & 0.7259 & 0.9248 \\
\hline 0.9 & 0.5098 & 0.8014 & 1.0206 & 0.5099 & 0.8015 & 1.0205 \\
\hline 1 & 0.5642 & 0.8862 & 1.1284 & 0.5642 & 0.8863 & 1.1284 \\
\hline
\end{tabular}

From this table it is obvious that the agreement between the present work and the previous published work is good. Again in order to conserve space the influence of the parameters considered in this study along with the parameter $\zeta$ on velocity, temperature, and concentration profiles will be described only without graphs. Furthermore, for some parameters the local Nusselt and Sherwood numbers will be described only. At a given value of the exponent $m$ (in Eq. 9) and for lower values of the non-similarity parameter $\zeta$ it was noticed that the velocity gradient is greater. At a fixed value of $\zeta$, as $m$ increases the velocity, temperature, and concentration gradients are larger and the momentum, thermal, and concentration boundary layer thicknesses are smaller. From the numerical results it was noticed for $\Gamma=M=\lambda=1, N=$ $2, L e=5$, and $S=0.3$ that the boost in the value of $m(m=0,0.5,1)$ lead to increase in the amount of heat and mass transfer.

To a given value of $\zeta$ the rise in the parameter of inertia lower the velocity of the fluid. Also, it was noticed that the velocity profiles for natural convection thin near the cylinder surface and thick far from the cylinder surface. Furthermore, at a given value of the mixed convection parameter an increase in the inertia parameter increases the temperature and concentration of the fluid. It is observed that for pure forced convection, increasing of inertia effect parameter has no effectiveness on the temperature and concentration profiles. When the inertia effect parameter enhances $(\Gamma=0.1,1,10)$ with $m=0.5, M=\lambda=1, N=2, L e=5$, and $S=0.3$ the local Nusselt and Sherwood numbers dwindling. This is explicit because inertia effectiveness tends to delay the momentum transmit in the boundary layer and hence lessen the heat and mass transfer. The curves according to the different values of inertia effect parameter are converge to single point in the forced convection frontier. We can infer from this behavior that inertia term has small weight in forced convection when used the Ergun's correlation.

Numerical solution presented that the increasing value of the square of the Hartmann number leads to increasing the velocity gradient at a given value of $\zeta$, while decreasing the temperature and concentration gradients. It can be interpreted the reduction in the velocity of the fluid is due to the resistance of the magnetic force named Lorentz force. The action of the magnetic force on the local Nusselt and Sherwood numbers are shown in Fig. 2 and Fig. 3 respectively. 


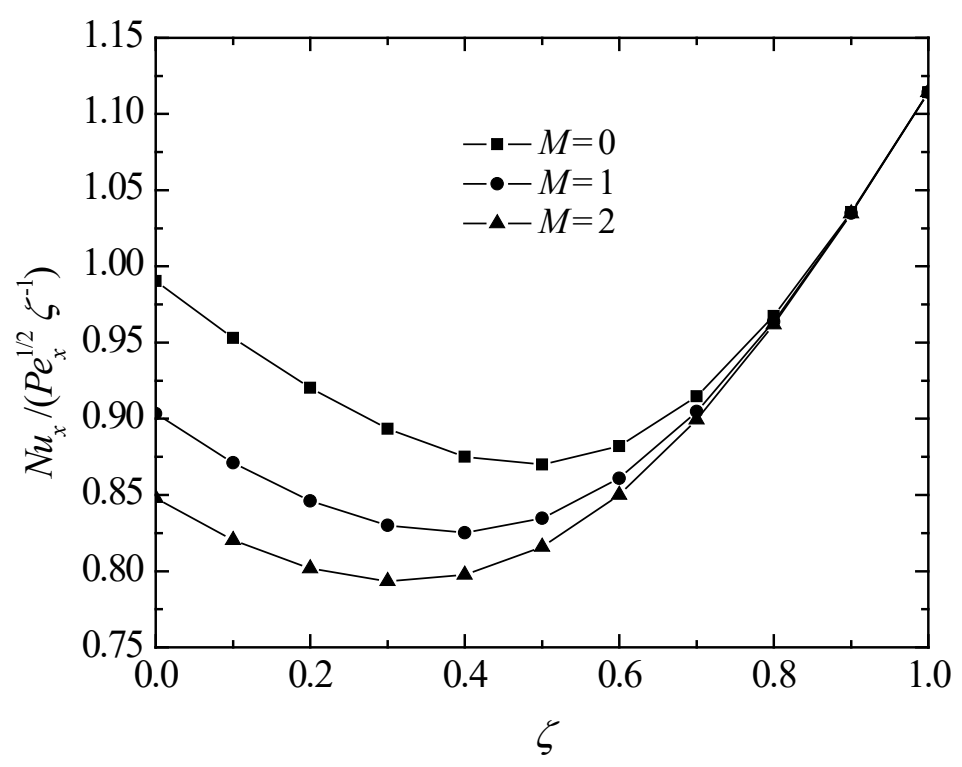

Fig. 2: Local Nusselt number variation for total regime of mixed convection with different values of square of the Hartmann number. $(m=0.5, \Gamma=\lambda=1, N=2$,

$$
L e=5, S=0.3)
$$

It is clear that the increasing of magnetic force causes lowering in the amount of heat and mass transfer. Also, this effect decay as we transfer to the forced convection dominated regime. The results presented that as the curvature parameter raises the velocity, temperature, and concentration gradient decreases. When $(\lambda=0.5,1,2)$ with $m=0.5, \Gamma=$ $M=1, N=2, L e=5$, and $S=0.3$ we can deduce that larger values of the curvature parameter give large amount of heat and mass transfer for whole region of mixed convection. This means that as the cylinder becomes slender, corresponding to a large value of $\left(x / r_{o}\right)$ would generate a higher heat and mass transfer rates.

The impact of buoyancy ratio parameter on the boundary layer profiles can be explained as follows. As the buoyancy ratio parameter excesses, the velocity gradient decreases and the temperature and

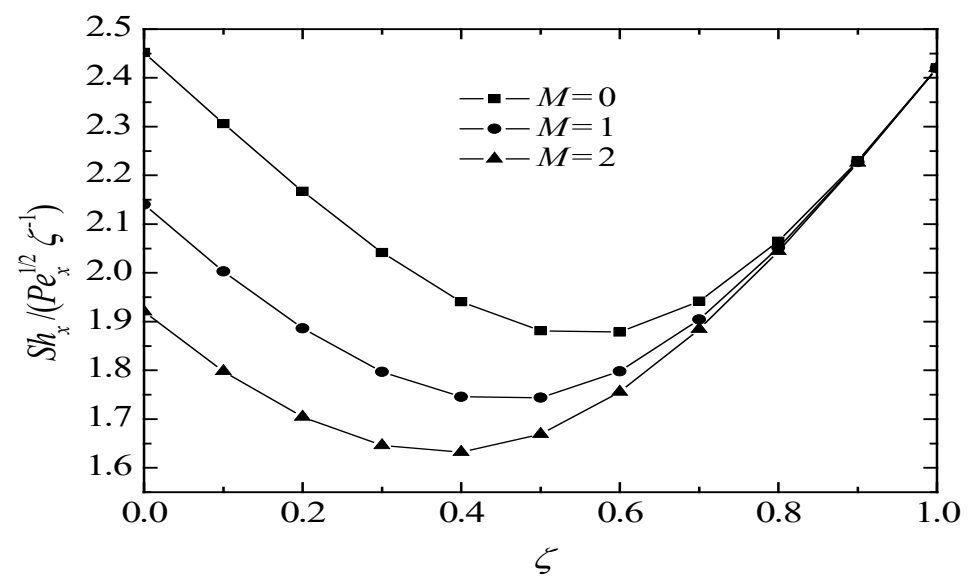

Fig. 3: Local Sherwood number variation for total regime of mixed convection with different values of square of the Hartmann number. $(m=0.5, \Gamma=\lambda=1$,

$$
N=2, L e=5, S=0.3)
$$


concentration gradients increases. Also it was observed that, for forced convection, the temperature and concentration are not influenced by the increase in the value of buoyancy ratio parameter. According to the above reasons the amount of heat and mass transfer enhances as the value of buoyancy ratio raise as presented in Fig. 4 and Fig. 5 respectively. It is clear that the effect of buoyancy ratio on heat and mass transfer is weak in the forced convection dominated regime.

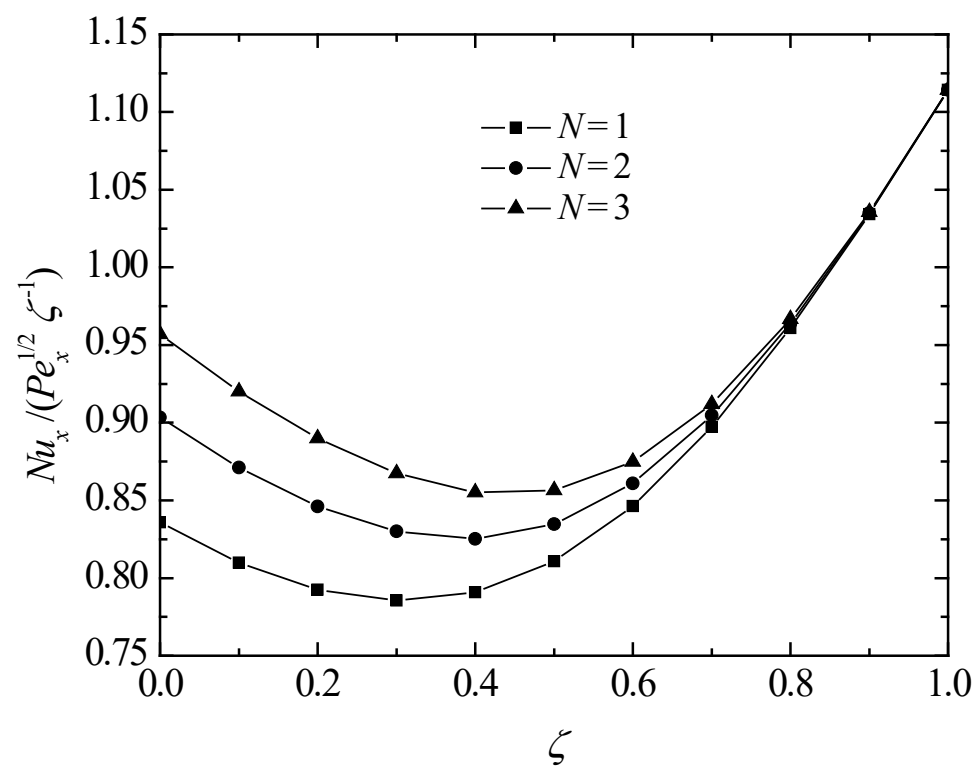

Fig. 4: Local Nusselt number variation for total regime of mixed convection with different values of buoyancy ratio. $(m=0.5, \Gamma=M=$ $\lambda=1, L e=5, S=0.3)$

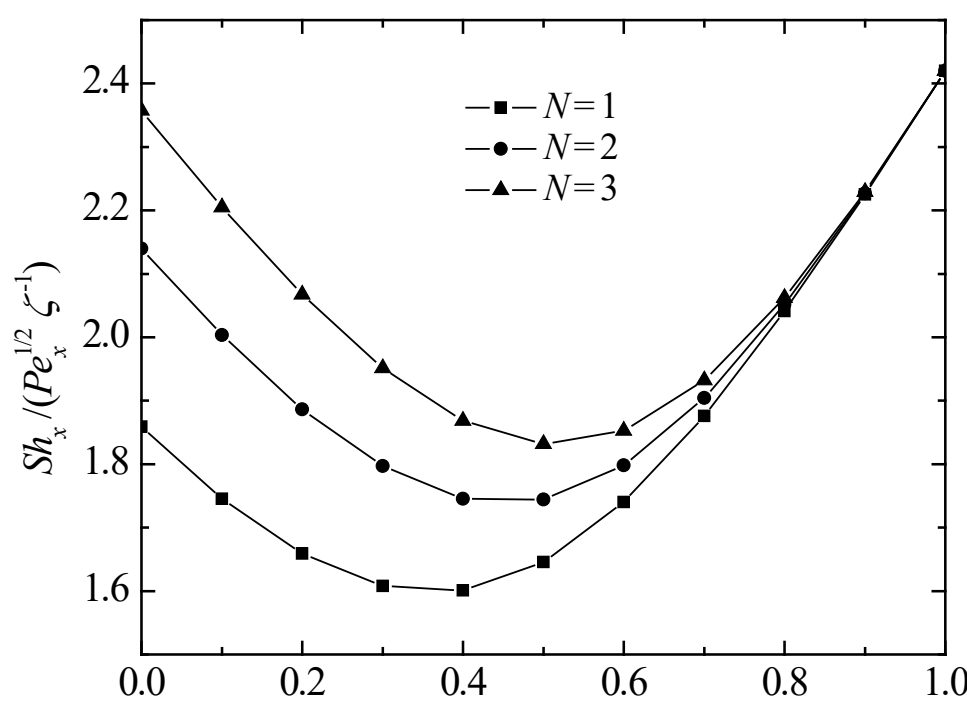

Fig. 5: Local Sherwood number variation for total regime of mixed convection with different values of buoyancy ratio. $(m=0.5, \Gamma=$ $M=\lambda=1, L e=5, S=0.3$ ) 
Increasing the value of the Lewis number has the effectiveness of increasing of velocity and concentration gradients while decreasing of temperature gradients. This is due to the fact that the rise in the thermal diffusivity lead to rise in the thermal buoyancy forces and consequently the temperature of the fluid raise. On the other hand due to the reduction in the mass diffusivity, the concentration buoyancy forces lowering and as a result the concentration of the fluid decrease. Fig. 6 indicates that the excess in the value of the Lewis number leads to reduction in the value of the local Nusselt number for lower and moderate values of local non-similarity parameter. For higher value of local non-similarity parameter (i.e. forced convection dominated region) this reduction can be neglected. On the contrary, Fig. 7 depicts

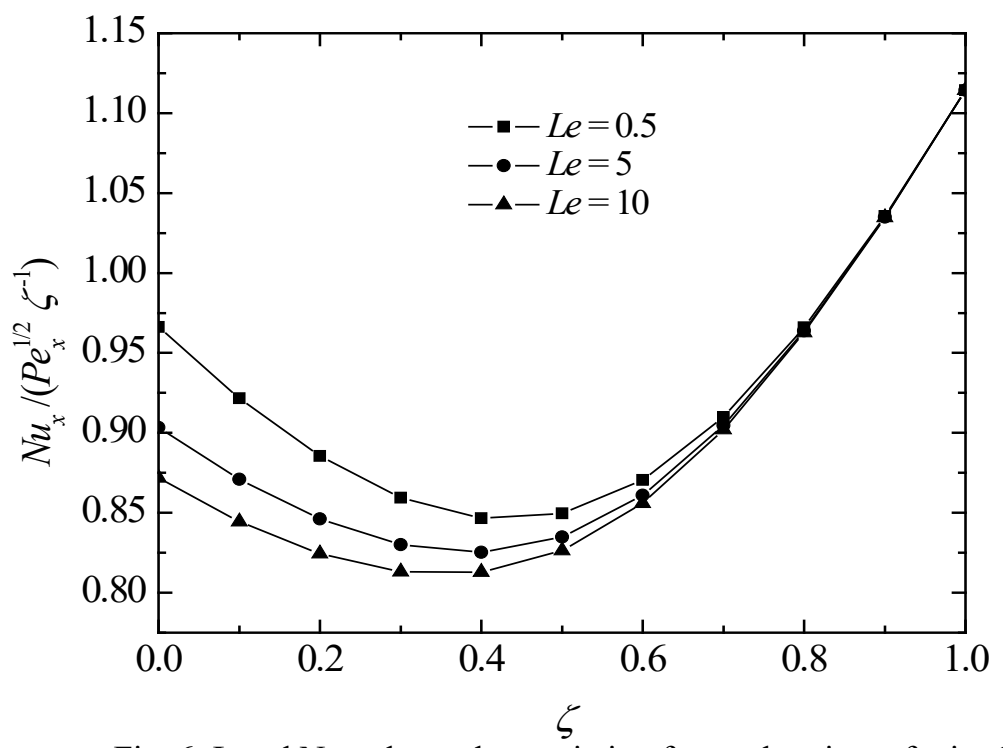

Fig. 6: Local Nusselt number variation for total regime of mixed convection with different values of the Lewis number. $(m=0.5, \Gamma=$

$$
M=\lambda=1, N=2, S=0.3 \text { ) }
$$

that the rise in the Lewis number leads to increase in the local Sherwood number for the total regime of mixed convection and this increase is appreciable.

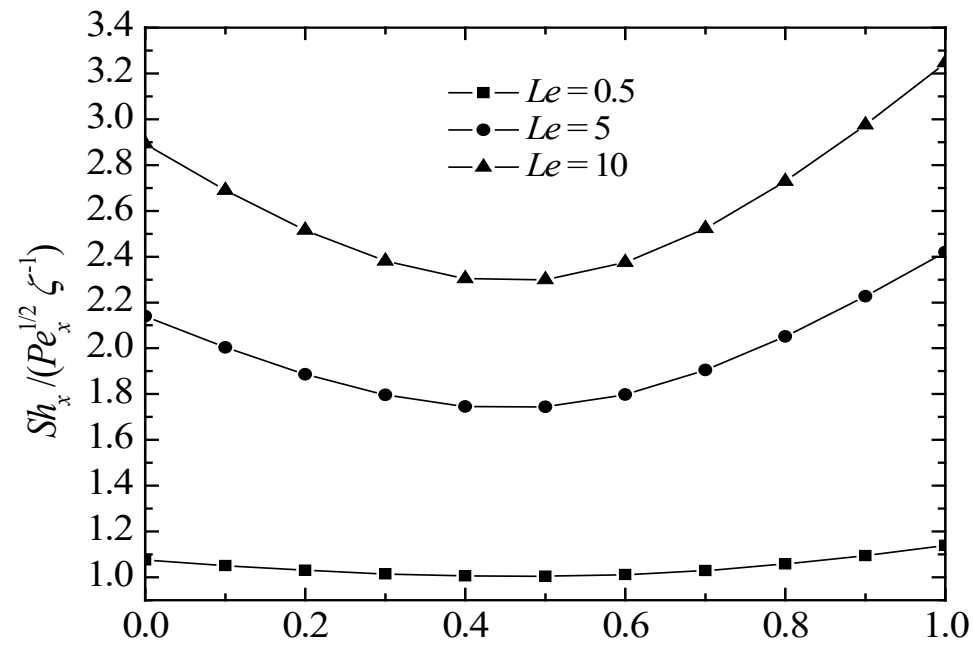

Fig. 7: Local Sherwood number Gariation for total regime of mixed convection with different values of the Lewis number. $(m=0.5, \Gamma=$ $M=\lambda=1, N=2, S=0.3)$ 
From the numerical results it was observed that the enhance of the thermal stratification parameter will increase the velocity, temperature, and concentration gradients and decrease the hydrodynamic, thermal, and concentration boundary layer thicknesses. Furthermore, when the stratification parameter growing the local Nusselt and Sherwood numbers decreases as shown in Fig. 8 and Fig. 9. Also from

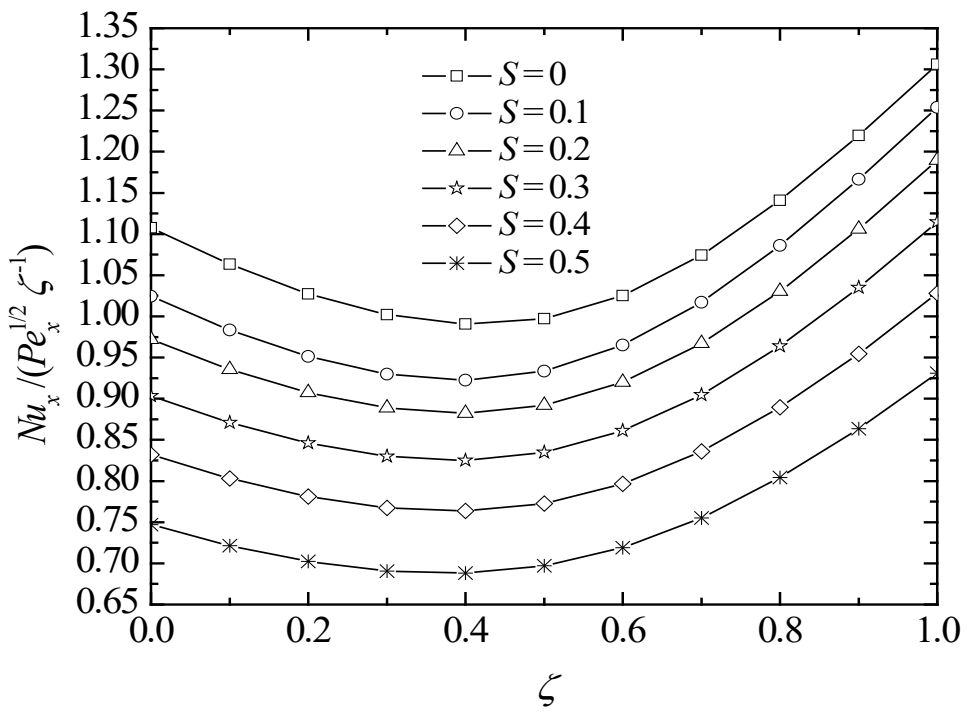

Fig. 8: Local Nusselt number variation for total regime of mixed convection with different values of local thermal stratification parameter. $(m=0.5, \Gamma=$ $M=\lambda=1, N=2, L e=5)$

Fig. 8 we can view that the entire regime of mixed convection is influenced significantly by the increasing of the stratification parameter. On the other hand, this action on the local Sherwood number is weak as compared with local Nusselt number, and can be restricted to natural convection and natural convection dominated region (lower values of mixed convection parameter). For forced convection dominated region this effect is very low and can be neglected as presented in Fig. 9.

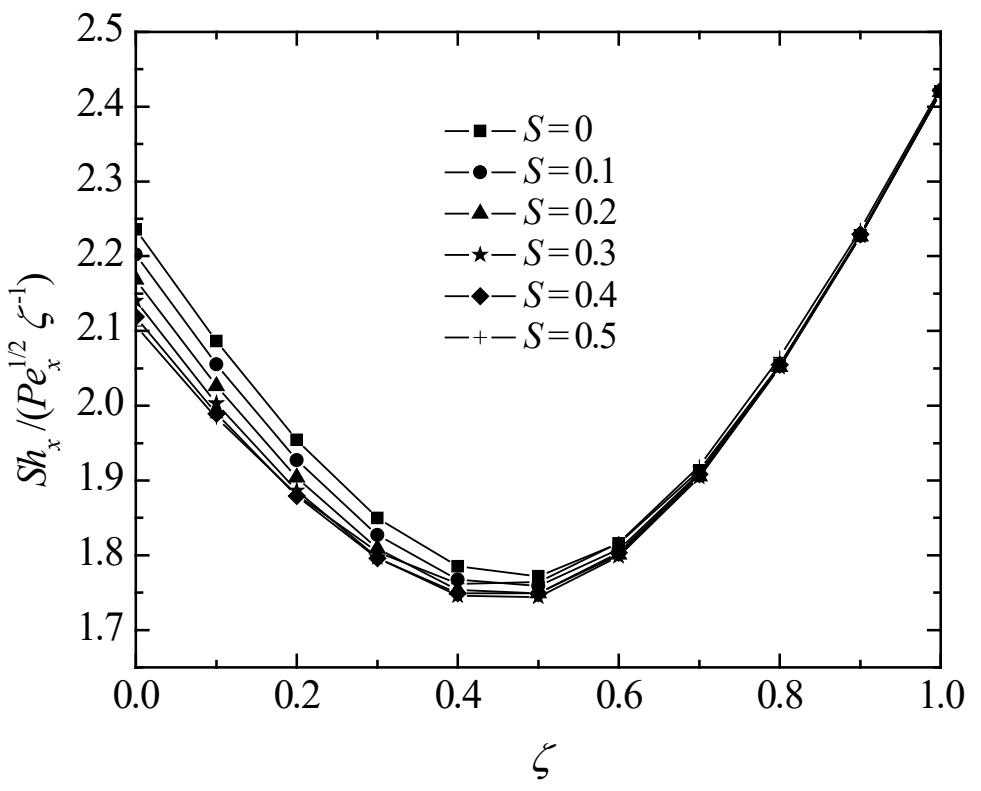

Fig. 9: Local Sherwood number variation for total regime of mixed convection with different values of local thermal stratification parameter.

$$
(m=0.5, \Gamma=M=\lambda=1, N=2, L e=5)
$$




\section{Conclusions}

The numerical results show that the raise in the value of $m$ lead to enhance the amounts of heat and mass transfer. When the inertia effect parameter growing, the local Nusselt and Sherwood numbers lessening. Increasing of magnetic force causes lowering amount of heat and mass transfer. Larger values of the curvature parameter would generate a higher heat and mass transfer rates. The amount of heat and mass transfer enhances as the value of buoyancy ratio parameter raise. The excess in the value of the Lewis number leads to reduction in the value of the local Nusselt number. On the contrary, the rise in the Lewis number leads to increase in the local Sherwood number. Finally, when the stratification parameter rises the local Nusselt and Sherwood number decreases.

\section{Acknowledgements}

The author would like to thank the college of engineering-university of Mosul for the support.

\section{References}

[1] C. - H. Chen and J. -H. Horng, "Natural Convection from a Vertical Cylinder in a Thermally Stratified Porous Medium," Heat and Mass Transfer, vol. 34, pp. 423-428, 1999.

[2] S. Mukhopadhyay and A. Ishak, "Mixed Convection Flow Along a Stretching Cylinder in a Thermally Stratified Medium," Hindawi Publishing Corporation, Journal of Applied Mathematics, vol. 2012, Article ID 491695, pp. 1-8, 2012.

[3] C. - H. Chen, "Mixed Convection Heat Transfer from a Horizontal Plate with Variable Surface Heat Flux in a Porous Medium," Heat and Mass Transfer, vol. 34, pp. 1-7, 1998.

[4] A. A. Afify, "Effects of Variable Viscosity on Non-Darcy MHD Free Convection Along a Non-isothermal Vertical Surface in a Thermally Stratified Porous Medium," Applied Mathematical Modelling, vol. 31, pp. 1621-1634, 2007.

[5] CH. Amanulla N. Nagendra and M. Suryanarayana Reddy, "Numerical Simulation of Slip Influence on Electric Conducting Viscoelastic Fluid Past an Isothermal Cylinder," Frontiers in Heat and Mass Transfer (FHMT), vol. 10, no. 10, pp. 1-13, 2018.

[6] M. Ganapathirao A. J. Chamkha and N. Srinivasa Rao, "Effects of Non-uniform Slot Suction/Injection and Chemical Reaction on Mixed Convective MHD Flow Along a Vertical Wedge Embedded in a Porous Medium," Frontiers in Heat and Mass Transfer (FHMT), vol. 13, no. 15, pp.1-13, 2019.

[7] J. H. Merkin and I. Pop, "Mixed Convection Boundary-Layer on a Vertical Cylinder Embedded in a Saturated Porous Medium," Acta Mechanica, vol. 66, pp. 251-262, 1987.

[8] M. Kumari G. Nath and I. Pop, "Non-Darcy Mixed Convection Flow with Thermal Dispersion on a Vertical Cylinder in a Saturated Porous Medium," Acta Mechanica, vol. 100, pp. 69-77, 1993.

[9] W. B. Hooper T. S. Chen and B. F. Armaly, "Mixed Convection Along an Isothermal Vertical Cylinder in Porous Media," Journal of Thermophysics and Heat Transfer, vol. 8, no. 1, pp. 92-99, 1994.

[10] T. K. Aldoss M. A. Jarrah and B. J. Al-Sha'er, "Mixed Convection from a Vertical Cylinder Embedded in a Porous Medium: Non-Darcy Model," Int. J. Heat Mass Transfer, vol. 39, no. 6, pp. 1141-1148, 1996.

[11] T. K. Aldoss, "MHD Mixed Convection from a Vertical Cylinder Embedded in a Porous Medium," Int. Comm. Heat Mass Transfer, vol. 23, no. 4, pp. 517-530, 1996.

[12] M. Kumari C. Bercea and I. Pop, "Effect of Non-uniform Suction or Injection on Mixed Convection Flow over a Vertical Cylinder Embedded in a Porous Medium," Malaysian Journal of Mathematical Sciences, vol. 1, no. 2, pp. 193-204, 2007.

[13] R. S. R. Gorla and A. Hossain, "Mixed Convection Boundary Layer Flow over a Vertical Cylinder Embedded in a Porous Medium Saturated with a Nanofluid," International Journal of Numerical Methods for Heat \& Fluid Flow, vol. 23, no. 8, pp. 1393-1405, 2013.

[14] A. M. Rohni S. Ahmad J. H. Merkin and I. Pop, "Mixed Convection Boundary-Layer Flow Along a Vertical Cylinder Embedded in a Porous Medium Filled by a Nanofluid,” Transp Porous Med, vol. 96, pp. 237-253, 2013. 
[15] B. Jafarian M. Hajipour and R. Khademi, "Conjugate Heat Transfer of MHD Non-Darcy Mixed Convection Flow of a Nanofluid over a Vertical Slender Hollow Cylinder Embedded in Porous Media," Trans. Phenom. Nano Micro Scales, vol. 4, no. 1, pp. 1-10, 2016.

[16] J. -J. Shu Q. -W. Wang and I. Pop, "Dual Solutions for Opposing Mixed Convection in Porous Media," Journal of Heat Transfer-Transactions of the ASME, vol. 139, no. 10, pp. 102-105, 2017.

[17] S. A. Mohammad, "Heat and Mass Transfer by Hydromagntic Mixed Convection Flow Along a Vertical Cylinder Embedded in a Non-Darcy Porous Medium with Heat Source," International Journal of Mechanical and Production Engineering Research and Development (IJMPERD), vol. 9, no. 5, pp. 171-182, 2019.

[18] S. A. Mohammad, "Effects of Variable Viscosity on Heat and Mass Transfer by MHD Mixed Convection Flow Along a Vertical Cylinder Embedded in a Non-Darcy Porous Medium," Frontiers in Heat and Mass Transfer (FHMT), vol. 14, no. 7, pp. 1-10, 2020.

[19] J. C. Hsieh T. S. Chen and B. F. Armaly, "Mixed Convection Along a Non-isothermal Vertical Flat Plate Embedded in a Porous Medium: The Entire Regime," Int. J. of Heat and Mass Transfer, vol. 36, no. 7, pp. 1819-1825, 1993. 